\title{
On the quantum criticality in the ground and the thermal states of XX model
}

\author{
W. Son ${ }^{1}$ and V. Vedral ${ }^{2,3,4}$ \\ ${ }^{1}$ Centre for Quantum Technologies, National University of Singapore, 3 Science Drive 2, Singapore 117543 \\ ${ }^{2}$ Centre for Quantum Technologies, National University of Singapore, 3 Science Drive 2, Singapore 117543 \\ ${ }^{3}$ The School of Physics and Astronomy, University of Leeds, Leeds, LS2 9JT, United Kingdom \\ ${ }^{4}$ Department of Physics, National University of Singapore, 2 Science Drive 3, Singapore 117542
}

(Dated: December 20, 2018)

\begin{abstract}
We compare the critical behavior of the ground state and the thermal state of the XX model. We analyze the full energy spectrum and the eigenstates to reconstruct the ground state and the thermally excited state. With the solutions, we discuss about several physical properties of the states, which are related to quantum phase transition, in various limits, at zero temperature as well as at a thermal equilibrium.

PACS numbers:
\end{abstract}

Conventional definition of quantum phase transition (QPT) is given by energy level crossing of ground energy state with varying external parameter [1]. The definition is based on the view that the ground energy state is taken as a quantum phase itself and, thus, QPT is a transition occurring at the point where a zero temperature state is transited from one state to the other. Because of the singular structure of the energy transition, the point of QPT is known to be detected by non-analyticity of free energy.

Within the definition, a difficulty may arise if one tries to consider a finite temperature QPT. It is because QPT is only defined at $T=0$. Typical treatment of finite temperature phase transition so far is provided by the non-analyticity of thermodynamic properties or the characteristic wave length of the system [2, 3]. However, it is not straightforward how the singularity in the thermal state is consistently combined with the notion of energy level crossing at zero temperature.

In this article, we study the full solutions of XX spin chain model, not only the energy eignevalues but also the eignestates in the original spin basis. With the solutions, we demonstrate how the sequential energy level crossings at zero temperature is led to a continuous QPT in infinite spin limit, $N \rightarrow \infty$. In the XX spin model, the number of ground energy level-crossings grow with the size of the chain. When $N \rightarrow \infty$, the crossing points become dense within a sharply defined critical region, giving rise to a continuous QPT. This is a typical example when a ground state is no longer in a pure state but in a mixed state. At the same time, each crossing point of the ground energy state becomes fully analytic.

The situation is comparable to a state in finite temperature which is a mixture of all the state spectrums with the Boltzmann distribution. We investigate the structure of state in a thermally excited XX spin chain and the purity of the state to compare it with the ground state of the infinite chain. In the case, it can be seen that the temperature is acting as a controlling parameter to determine the degree of state mixture in the sys- tem. Thermodynamic properties of the thermal state are remained analytic in the region. As temperature is increased, the state moves into a thermal mixture which destroys the quantum correlations, entanglement, in the system. Extensive discussions on the relation between QPT and entanglement in the XX model can also be found at [4, [5, 8].

\section{MODEL AND FULL SOLUTIONS}

We start our discussion by considering $N$ spin $1 / 2$ particles in a line, coupled by nearest neighbor XX interaction, with Hamiltonian

$$
H=-\left[\sum_{i=1}^{N} \frac{J}{2}\left(\sigma_{i}^{x} \sigma_{i+1}^{x}+\sigma_{i}^{y} \sigma_{i+1}^{y}\right)+B \sigma_{i}^{z}\right]
$$

where we have assumed that the exchange interaction $J$ fixes the energy unit. The model with periodic boundary condition has been firstly solved by Katsura [6] and recently discussed at finite size in Ref. 7]. Here, we assume open boundaries, with $\sigma_{N+1}=0$. After Jordan-Wigner (JW) and Fourier transformations,

$$
d_{k}=\sqrt{\frac{2}{N+1}} \sum_{l=1}^{N} \sin \left(\frac{\pi k l}{N+1}\right) \bigotimes_{m=1}^{l-1} \sigma_{m}^{z} \sigma_{l}^{-},
$$

the Hamiltonian takes a diagonal form, $H=$ $\sum_{k=1}^{N} \Lambda_{k} d_{k}^{\dagger} d_{k}+N B$ where $\Lambda_{k}=-2 B+$ $2 \cos [(\pi k) /(N+1)]$. The $2^{N}$ eigenenergies are $\epsilon_{l} \equiv \sum_{k=1}^{N} \Lambda_{k}\left\langle\psi_{l}\left|d_{k}^{\dagger} d_{k}\right| \psi_{l}\right\rangle+N B$ and the corresponding eigenstates are found

$$
\left|\psi_{l}\right\rangle=\prod_{k=1}^{N}\left(d_{k}^{\dagger}\right)^{\alpha_{k}^{(l)}}|\Omega\rangle,
$$

where the binary integer number $\alpha_{k}^{(l)} \in\{0,1\}$ identifies eigenenergy as $\epsilon_{l} \equiv \sum_{k=1}^{N} \Lambda_{k} \alpha_{k}^{(l)}+N B$. The state $|\Omega\rangle$ is 
(a)

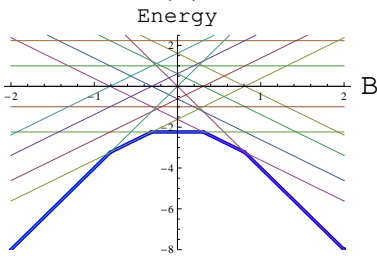

(b)

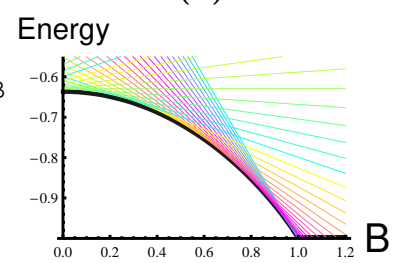

FIG. 1: (a) The spectrum of $2^{4}$ energy levels for the $N=4$ $\mathrm{XX}$ spin chains. The energies vary as a function of magnetic field $B$. Blue lines are the energy change of the ground state. Energy level crossings occur at the critical points in the ground state as well as in excited states. Ground state entanglement is jumping as the state is switched from an entangled state to another state. (b) The ground state energy per spin against the magnetic field $B$ in the thermodynamic limit. The energy is plotted together with large spin chain case, $N=50$. The continuous energy level crossings occur in the limit at the region $0<B<1$.

the vacuum: $d_{k}|\Omega\rangle=0 \forall k$. It is also useful to know that $\left\langle\psi_{i} \mid \psi_{j}\right\rangle=\delta_{i, j}$. The energy diagram of $\epsilon_{l}$ for $N=4$ as a function of $B$ is illustrated in Fig 1 (a).

\section{GROUND STATE OF XX SPIN CHAIN}

In this section, we discuss about the ground state of the $\mathrm{XX}$ model. The ground energy state is the lowest energy state which is a function of the transverse magnetic field $B$. Letting $B_{k}=\cos [k \pi /(N+1)]$ and defining the $k$-th region, $B_{k+1}<B<B_{k}$, we can find the ground state $\left|\psi_{g}^{k}\right\rangle=d_{k} d_{k-1} \cdots d_{1}\left|\psi_{g}^{0}\right\rangle=\prod_{l=k+1}^{N} d_{l}^{\dagger}|\Omega\rangle$ and its energy as $\epsilon_{g}^{k}=-(N-2 k) B-2 \sum_{l=1}^{k} \cos \left(\frac{\pi l}{N+1}\right)$ (refer [5] for detail). The index $k$ is an integer in $1 \leq k<N$ and $B_{k}$ represents the points where energy level crossings occur. When $B<B_{N}$ or $B>B_{1}$, no other crossing exists and the ground state is simply given by the fermion vacuum state. The ground state energy for $N=4$ is plotted in Fig. 1 (a) by blue lines.

The ground state in the spin bases after $k$ level crossing can be obtained recursively $\left|\varphi_{g}^{k}\right\rangle=$ $\prod_{k^{\prime}=1}^{k}\left[\sum_{l=1}^{N} S_{l}^{k^{\prime}}\left(\prod_{m=1}^{l-1} \sigma_{m}^{z} \sigma_{l}^{-}\right)\right]|\uparrow\rangle^{\otimes N}$ where $S_{l}^{k} \equiv$ $\sqrt{2 /(N+1)} \sin [(\pi k l) /(N+1)]$ and the explicit form of the state is given as

$$
\left|\varphi_{g}^{k}\right\rangle=\left(\frac{2}{N+1}\right)^{\frac{k}{2}} \sum_{l_{1}<l_{2}<\cdots<l_{k}} C_{l_{1} l_{2} \cdots l_{k}}\left|l_{1}, l_{2}, \cdots, l_{k}\right\rangle
$$

where $\left|l_{1}, l_{2}, \cdots, l_{k}\right\rangle$ is the state whose $l_{1}$-th, $l_{2^{-}}$ th, $\cdots, l_{k}$-th indicate the position of flipped spin. The amplitudes in the state are $C_{l_{1} l_{2} \cdots l_{k}}=$ $\sum_{P}(-1)^{P} \sin \left(\frac{\pi P(1) l_{1}}{N+1}\right) \sin \left(\frac{\pi P(2) l_{2}}{N+1}\right) \cdots \sin \left(\frac{\pi P(k) l_{k}}{N+1}\right)$.

Here, the sum extends over all the permutation of the numbers from 1 to $\mathrm{k}$, (denoted by $P(k)$ ). We point out that, at each crossing point (caused by the variation of $B)$, the ground state jumps discontinuously from one symmetric subspace to the another, orthogonal to the previous one. To see the state explicitly, we illustrate the ground states for $N=4$ in spin bases which are

$$
\begin{aligned}
& \left|\varphi_{g}^{0}\right\rangle=|\uparrow, \uparrow, \uparrow, \uparrow\rangle, \quad\left|\varphi_{g}^{1}\right\rangle=a_{1}^{-}|\downarrow, \uparrow, \uparrow, \uparrow\rangle+a_{1}^{+}|\uparrow, \downarrow, \uparrow, \uparrow\rangle+a_{1}^{+}|\uparrow, \uparrow, \downarrow, \uparrow\rangle+a_{1}^{-}|\uparrow, \uparrow, \uparrow, \downarrow\rangle \\
& \left|\varphi_{g}^{2}\right\rangle=a_{2}(|\downarrow, \downarrow, \uparrow, \uparrow\rangle+2|\uparrow, \downarrow, \downarrow, \uparrow\rangle+2|\downarrow, \uparrow, \uparrow, \downarrow\rangle+|\uparrow, \uparrow, \downarrow, \downarrow\rangle+\sqrt{5}|\downarrow, \uparrow, \downarrow, \uparrow\rangle+\sqrt{5}|\uparrow, \downarrow, \uparrow, \downarrow\rangle) \\
& \left|\varphi_{g}^{3}\right\rangle=a_{3}^{-}|\uparrow, \downarrow, \downarrow, \downarrow\rangle+a_{3}^{+}|\downarrow, \uparrow, \downarrow, \downarrow\rangle+a_{3}^{+}|\downarrow, \downarrow, \uparrow, \downarrow\rangle+a_{3}^{-}|\downarrow, \downarrow, \downarrow, \uparrow\rangle, \quad\left|\varphi_{g}^{4}\right\rangle=|\downarrow, \downarrow, \downarrow, \downarrow\rangle
\end{aligned}
$$

where $a_{1}^{ \pm}=-a_{3}^{ \pm}=\frac{1}{2} \sqrt{1 \pm \frac{1}{\sqrt{5}}}$ and $a_{2}=-\frac{1}{2 \sqrt{5}}$. From the state, it is clear that the superscript of $\varphi$ indicates the number of down spins. The ground state at the $k$-th region is composed of ${ }_{N} C_{k}=N ! /[k !(N-k) !]$ orthogonal vectors in spin bases which are the possible choices of $\mathrm{k}$ spins, out of total $\mathrm{N}$ spins. The ground states are in an orthogonal subspace, $\left\langle\varphi_{g}^{k} \mid \varphi_{g}^{k^{\prime}}\right\rangle=\delta_{k, k^{\prime}}$.

It is found that the ground energy spectrum and the ground state become continuous at the thermodynamic limit, $N \rightarrow \infty$. In the limit, the sum in $\epsilon_{g}^{k}$ turns into a definite integral and thus the ground state energy reads

$$
\lim _{N \rightarrow \infty} \frac{\epsilon_{g}(B)}{N}=\frac{2}{\pi}\left[B\left(\arccos B-\frac{\pi}{2}\right)-\sqrt{1-B^{2}}\right] .
$$

Within the critical region the ground state energy is analytic everywhere except for $B= \pm 1$. From the finite size analysis we observe that such a critical line becomes a dense set of level crossing (see Fig. 1 (b)) and therefore is possible to be considered as a line of continuous QPT. At the crossing points $B_{k}$, the state exists as a mixed state with equal mixture of the states in a neighboring 
$k$-th region, $\left|\varphi_{g}^{k}\right\rangle$ and $\left|\varphi_{g}^{k+1}\right\rangle$, i.e.

$$
\rho\left(B_{k}\right)=\frac{1}{2}\left(\left|\varphi_{g}^{k}\right\rangle\left\langle\varphi_{g}^{k}|+| \varphi_{g}^{k+1}\right\rangle\left\langle\varphi_{g}^{k+1}\right|\right) .
$$

Therefore, one can find that the state in the thermodynamics limit is also varying from a mixed state into the other mixed state continuously as a function of $B$. That is because the critical points $B_{k}$ is now continuous function, $B_{\omega}=\lim _{N \rightarrow \infty} \cos (k \pi /(N+1))=\cos (\pi \omega)$ where $0<\omega<1$. Moreover, one would find that the mixedness of the state in the limit is remained as a constant for any $B$ in $-1<B<1$ as $\operatorname{Tr} \rho(B)^{2}=1 / 2$. The mixedness (or purity) $1 / 2$ of the ground state in the limit can also be proved from the limiting case of a thermal state which will be discussed in the following section.

\section{SPIN CHAIN IN THERMAL EQUILIBRIUM}

In this section, we investigate the state in a thermal equilibrium and discuss about the properties related with quantum phase transition. At a finite temperature, the state exists in a mixture of all the energy state distributed by Boltzmann statistics. The state in a diagonal basis $\left|\psi_{l}\right\rangle=\Pi_{k=1}^{N}\left(d_{k}^{\dagger}\right)^{\alpha_{k}^{(l)}}|\Omega\rangle, \alpha_{k}^{(l)} \in\{0,1\}$ is given as

$$
\rho_{T}=\sum_{l=1}^{2^{N}} p_{l}\left|\psi_{l}\right\rangle\left\langle\psi_{l}\right|
$$

where $p_{l}=\exp \left(-\beta \epsilon_{l}\right) / Z$ is the Boltzmann distribution of the thermal state with the partition function $Z=e^{-\beta N B} \prod_{k=1}^{N}\left(1+e^{-\beta \Lambda_{k}}\right)$. In terms of the spin basis, the thermal state further can be decomposed into the states which are in a symmetric subspace as,

$$
\rho_{T}=\sum_{m=0}^{N} \sum_{r=1}^{N C_{m}} q_{r}^{m}\left|\varphi_{r}^{m}\right\rangle\left\langle\varphi_{r}^{m}\right| .
$$

The index $m$ in the superscript indicates a symmetric subspace where vector components of the state contain the $m$ number of spins flipped and the subscript index $r$ identifies the ${ }_{N} C_{m}=N ! /[m !(N-m) !]$ orthogonal vectors within the subspace. Thus, the state satisfies the orthonormal condition, $\left\langle\varphi_{r}^{m} \mid \varphi_{r^{\prime}}^{m^{\prime}}\right\rangle=$ $\delta_{r^{\prime}, r} \delta_{m^{\prime}, m}$. The state $\left|\varphi_{r}^{m}\right\rangle$ can be obtained from the $l$ th state $\left|\psi_{l}\right\rangle$ using the transformation in (2), $\left|\varphi_{r}^{m}\right\rangle=$ $\prod_{k=1}^{N}\left[\sum_{j=1}^{N} S_{j}^{k}\left(\prod_{i=1}^{j-1} \sigma_{i}^{z} \sigma_{j}^{-}\right)\right]^{\alpha_{k}^{(l)}}|\uparrow\rangle^{\otimes N}$ where $S_{j}^{k} \equiv$ $\sqrt{2 /(N+1)} \sin [(\pi j k) /(N+1)]$ and $\alpha_{k}^{(l)} \in\{0,1\}$. The values of the label $l$ for the binary vectors $\vec{\alpha}_{l}=\sum_{k} \alpha_{k}^{(l)} \hat{v}_{k}$ are determined by $r$ and $m$ through an index match $l=r+\sum_{s=0}^{m-1}{ }_{N} C_{s}$ under the constraints $\sum_{k=1}^{N} \alpha_{k}^{(r)}=m$. (It starts from $l=1$ when $(r, m)=(1,0)$.) Similarly, the probabilities of the Boltzmann distribution are given as $p_{l} \equiv q_{r}^{m}=\exp \left(-\beta \epsilon_{r}^{m}\right) / Z$. The lowest energy state for a fixed $m$ become a ground state at a given region of $B$, i.e $\left|\varphi_{1}^{m}\right\rangle \equiv\left|\varphi_{g}^{m}\right\rangle$. Furthermore, the corresponding energy eigenvalues by the subspace indexes $m$ and $r$ can be found as

$$
\epsilon_{l} \equiv \epsilon_{r}^{m}=-(N-2 m) B-2 \sum_{k=1}^{N} \cos [\pi k /(N+1)] \alpha_{k}^{(r)} .
$$

This shows that the slop of the energy against $B$ is invariant for the states in a given symmetric subspace, with a fixed $m$, and, thus, it also gives a proof that the energy level crossings of excited states occur only between the states in a different symmetric subspace. The level crossings of the excited states for $N=4$ spins also can be seen in Fig. 11(a). (a)

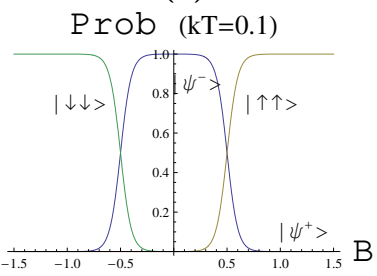

(b)

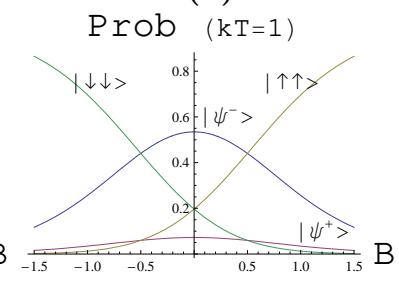

FIG. 2: Distributions of thermal state population for the $N=2 \mathrm{XX}$ spin chain when (a) $k T=0.1$ and (b) $k T=1$. The states $\left|\psi^{-}\right\rangle$and $\left|\psi^{+}\right\rangle$are the singlet and the triplet. Critical behavior disappears as the system goes into a thermal equilibrium.

As a special case, we inspect the density matrix of the thermally excited state when $N=2$ which is in a form,

$$
\rho_{T}^{N=2}=p_{1}|\uparrow, \uparrow\rangle\left\langle\uparrow, \uparrow\left|+p_{2}\right| \psi^{-}\right\rangle\left\langle\psi^{-}\left|+p_{3}\right| \psi^{+}\right\rangle\left\langle\psi^{+}\left|+p_{4}\right| \downarrow, \downarrow\right\rangle\langle\downarrow, \downarrow|
$$

where $\left|\psi^{ \pm}\right\rangle=\frac{1}{\sqrt{2}}(|\uparrow, \downarrow\rangle \pm|\downarrow, \uparrow\rangle)$. The state is expended in the three different symmetric subspaces, where there is no spin flip $(\mathrm{m}=0)$, one spin flip $(\mathrm{m}=1)$ and two spin flips $(\mathrm{m}=2)$. Each subspace contains ${ }_{2} C_{0},{ }_{2} C_{1}$ and ${ }_{2} C_{2}$ orthogonal elements of states, $\{|\uparrow, \uparrow\rangle\},\left\{\left|\psi^{-}\right\rangle,\left|\psi^{+}\right\rangle\right\}$and $\{|\downarrow, \downarrow\rangle\}$. We plot the probability distribution of the 
states $p_{l}$ in Fig 2. One would find that the distribution and the partition function become analytic for any $B$ and $T$ as $T$ is away from zero. If we investigate entanglement in the system, one would identify that the state become separable when $4 p_{1} p_{4}>\left(p_{2}-p_{3}\right)^{2}$, (from the negativity [9], the negative eigenvalue of partially transposed state), leading to a critical condition for temperature $k T>1.13459$. Interestingly, the separability condition for the two qubit thermal state is independent of the strength of magnetic field $B$ except when $T=0$. Thus, the singular behavior of entanglement with respect to $B$ is washed out as the temperature increased. This also has been depicted by the purity of the two qubit system in Fig. 3. (a)

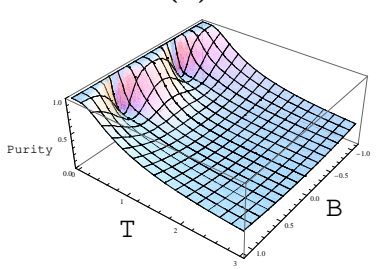

(b)

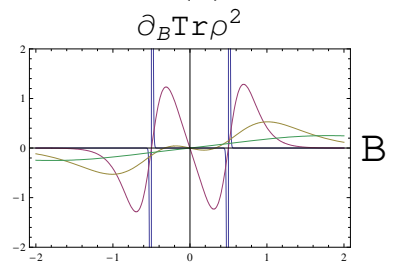

FIG. 3: (a) Purity of two qubit system as a function of magnetic field $B$ and temperature $T$. (b) Derivatives of the purity as a function of $B$ at different temperatures, $T=0.01$ (blue), $T=0.3$ (pink), $T=0.8$ (yellow) and $T=2$ (green). At a low temperature region, the purity of the system shows peaks near the critical points $B= \pm 1 / 2$. This is the point where the derivative of purity w.r.t. B becomes singular when $T=0$. At the high temperature region, the purity becomes monotonic convex function of $\mathrm{B}$ and the derivative of the purity approaches to a linear function.

With the density matrix of the thermal state (8), we investigate a physical property which may be directly related to the critical behavior of the system. The mixedness of the thermal state for any $N$ is

$$
\operatorname{Tr} \rho_{T}^{2}=\sum_{l} p_{l}^{2}=\prod_{k=1}^{N}\left[1-\left(1+\cosh \beta \Lambda_{k}\right)^{-1}\right] .
$$

This characterizes how far the state is from a pure state. The purity is plotted in Fig 3 for a small number of qubits $(N=2)$ and for a large number of qubits $(N=10)$ in

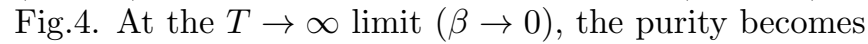
$1 / 2^{N}$. Thus, in the high temperature region and the thermodynamic limit, $N \rightarrow \infty$, the purity approaches to zero where the state is in a complete mixture of infinitely many orthogonal states. At the opposite extreme, $T \rightarrow 0$, the purity becomes either 1 or $1 / 2$ depending on the $B$ value. It is $1 / 2$ when $B$ is $\cos [k \pi /(N+1)]$ and 1 otherwise. When $N \rightarrow \infty$, the purity becomes $1 / 2$, i.e. $\lim _{N \rightarrow \infty} \lim _{T \rightarrow 0} \operatorname{Tr} \rho_{T}^{2}=1 / 2$ in $-1<B<1$ because there exits at least a single value of $B$ in the interval to make $\Lambda_{k}=0 \exists k$. In the $|B|>1$ region, the purity is
1. This proves that the ground state of the infinite $\mathrm{XX}$ chain in the region $|B|<1$ is the equal mixture of the neighboring states as (7). Furthermore, it is straightforward to see that entanglement disappears in the region $|B|>1$ and at high temperature. The separability of infinite XX chain with entanglement witness has been fully treated in [8].

In our analysis of the XX spin chain, it has been shown that the fundamental difference between the thermal state and the ground state of the infinite chain is only the degree of state mixedness. In both of the cases, the changes of states are impossible to be detected by singularity due to the continuity of state changes. In that sense, it can be pointed out that the energy level crossing is a particular mode of the ground state change when the state is in a pure state. In general, the state change can be detected by the change of entanglement, up to local unitary, which is destroyed by mixedness of the states. In fact, entanglement identifies the true quantum phase (or state) of a system since it is the property which can exist only in quantum systems whether they are pure or mixed 10]. Our approaches are general enough to be used for the discussions about the QPT and related properties of any other many particle systems.

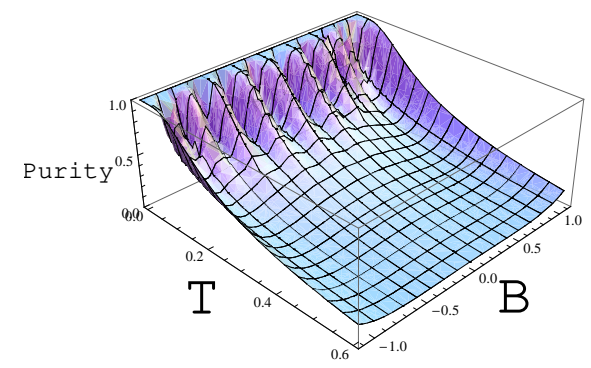

FIG. 4: Plot of the purity as a function of $B$ and $T$ when $N=10$. The state is in a pure state when $\operatorname{Tr} \rho^{2}=1$ and is in a completely mixed state when $\operatorname{Tr} \rho^{2}=1 / 2^{10}$. The purity of state is singular at the critical points of $B$ where the state exists as a mixture of two orthogonal symmetric states. It becomes smaller as $T$ is increased.

Acknowledgement- This work is supported by the National Research Foundation \& Ministry of Education, Singapore.

[1] S. Sachdev, Quantum Phase Transitions, Cambridge University Press (1999).

[2] S. Chakravarty, B. I. Halperin and D. R. Nelson, Phys. Rev. B 39, 2344(1989).

[3] S. Sachdev and A. P. Young, Phys. Rev. Lett. 78, 2220 (1997).

[4] L. Amico, R. Fazio, A. Osterloh and V. Vedral, Rev. Mod. Phys. 80, 517 (2008). 
[5] W. Son, L. Amico, F. Plastina and V. Vedral, arXiv:0807.1602 (2008).

[6] S. Katsura, Phys. Rev. 127, 1508 (1962).

[7] A. De Pasquale et al., Eur. Phys. J. Special Topics 160, 127 (2008), see also arxiv: 0801.1394 (2008).

[8] C. Brukner, V. Vedral, quant-ph/0406040 (2004); J. Hide, W. Son, I. Lawrie and V. Vedral, Phys. Rev. A
76, 022319 (2007).

[9] A. Peres, Phys. Rev. Lett. 77, 1413 (1996); M. Horodecki, P. Horodecki and R. Horodecki, Phys. Rev. A 223, 1 (1996).

[10] R. F. Werner, Phys. Rev. A 40, 4277 (1989). 